\title{
A flare of Still's disease following COVID-19 vaccination in a 34-year-old patient
}

\author{
Young Hun Jeon ${ }^{1} \cdot$ Doo-Ho Lim ${ }^{1}$ S Seung Won Choi ${ }^{1} \cdot$ Su Jin Choi ${ }^{1}$ (])
}

Received: 31 August 2021 / Accepted: 6 November 2021 / Published online: 19 November 2021

(c) The Author(s), under exclusive licence to Springer-Verlag GmbH Germany, part of Springer Nature 2021

\begin{abstract}
Vaccination is a cornerstone for reducing the risk of COVID-19 infection during a pandemic. Although the currently used COVID-19 vaccine is considered safe, some concerns persist regarding the likelihood of flares of rheumatic diseases. Still's disease is a rare auto-inflammatory disorder of unknown etiology, and the data on the flare of Still's disease following COVID-19 vaccination are limited. Therefore, we hereby present the case of a 34-year-old female patient with Still's disease who experienced a flare after a ChAdOx $1 \mathrm{nCoV}-19$ vaccination. The patient visited the emergency department complaining of fever, arthralgia, myalgia, pleuritic chest pain and macular salmon-pink rash on her back for the past 2 days. She had maintained low Still's disease activity with etanercept and low-dose glucocorticoid for 14 years. She received the ChAdOx $1 \mathrm{nCoV}-19$ vaccine 7 days before the flare. Laboratory investigations revealed leucocytosis and elevated serum levels of erythrocyte sedimentation rate, C-reactive protein, and ferritin. Computed tomography showed no specific findings. She received methylprednisolone pulse therapy, etanercept, and methotrexate for treating the Still's disease flare. However, her symptoms were not fully controlled, and she developed pericarditis, pleuritis, fever and macular rashes expanding to her extremities. After excluding infectious conditions by blood culture and pleural fluid analysis, we administered tocilizumab with methotrexate and prednisolone. Her symptoms and laboratory findings improved significantly, and she was discharged without symptoms 7 days later. Although rare, this case of a patient with Still's disease undergoing a flare following vaccination suggests that close observation of disease activity is warranted following COVID-19 vaccination.
\end{abstract}

Keywords Coronavirus · COVID-19 · Vaccination · Juvenile idiopathic arthritis

\section{Introduction}

In the face of the COVID-19 pandemic, newly developed vaccines are effectively preventing COVID-19 infections worldwide. Patients with rheumatic diseases are more susceptible to infection and are likely to experience more severe infections due to underlying autoimmune conditions and due to having received immunosuppressant therapy. Furthermore, COVID-19 infection itself may also precipitate flares of rheumatic diseases. Therefore, the European Alliance of Associations for Rheumatology (EULAR) recommended COVID-19 vaccination for patients with rheumatic diseases

Su Jin Choi

tetox2@hanmail.net

1 Division of Rheumatology, Department of Internal Medicine, Ulsan University Hospital, University of Ulsan College of Medicine, 877 Bangeojinsunhwan-doro, Dong-gu, Ulsan 44033, South Korea
[1]. On the other hand, several trials have reported on the safety of vaccines, and the association between disease flare and vaccination has also been an important issue. Recent preliminary data on post-market safety profile indicated that vaccines against COVID-19 do not necessarily exacerbate disease activity in rheumatic diseases [2]. Although the rapid development of vaccines has raised concerns regarding disease flare, the data reported so far are reassuring as far as the possibility of vaccine-related flares is concerned.

Nevertheless, there have been few reports of flares in patients with rheumatic diseases following vaccination for other diseases such as influenza and hepatitis B [3, 4]. Regarding COVID-19, disease flares following vaccination have been reported in the context of preexisting rheumatoid arthritis, IgA nephropathy, leukocytoclastic vasculitis, erythema nodosum, and psoriasis [5-9].

Still's disease is a rare, systemic, auto-inflammatory disorder characterized by spiking fever, skin rash, and arthritis. This disorder is classified based on onset, whether it occurs 
during the juvenile or adult stages. The former, juvenileonset Still's disease, is also known as a systemic form of juvenile idiopathic arthritis (JIA). Similar to the pathogenesis of COVID-19 infection, inflammatory cytokines such as IL-1 play an important role in Still's disease. Although it may be challenging to clarify the causal relationship between vaccination and disease flares, this case of Still's disease exacerbation following COVID-19 vaccination may provide information for appropriate recognition and treatment of Still's disease flares. However, there are still limited prior data on flares of Still's disease following COVID-19 vaccination. Accordingly, we report this case of a 34-yearold patient with Still's disease who experienced a flare following ChAdOx $1 \mathrm{nCoV}-19$ vaccination.

\section{Case report}

A 34-year-old woman visited our emergency department with a febrile sensation for 2 days. She also complained of arthralgia, myalgia, sore throat, pleuritic chest pain, and a macular salmon-pink rash on her back. The patient was diagnosed with Still's disease at the age of 12 years, and was doing well without a flare for 14 years on etanercept $50 \mathrm{mg}$ weekly and low-dose prednisolone. She received the first dose of ChAdOx $1 \mathrm{nCoV}-19$ vaccine 9 days ago.

On physical examination, we noticed a transient macular salmon-pink rash on her back during febrile periods. Her right elbow was swollen and warm. She had no pharyngeal injection, and her lung sounds were clear. Her vital signs were as follows: blood pressure, $156 / 105 \mathrm{mmHg}$; heart rate, 115 beats/min; body temperature, $37.8{ }^{\circ} \mathrm{C}$; and respiratory rate, 20 breaths/min. Initial laboratory tests indicated leucocytosis (WBC count 25,890/uL), anemia (Hemoglobin $11.7 \mathrm{~g} / \mathrm{dL}$ ), elevated erythrocyte sedimentation rate (ESR) $57 \mathrm{~mm} / \mathrm{hr}$ and serum levels of C-reactive protein (CRP) $13.96 \mathrm{mg} / \mathrm{dL}$. Anti-nuclear antibody was negative. Cervical, thoracic, abdominal, and pelvic computed tomography (CT) revealed no specific abnormalities. Blood cultures and real-time polymerase chain reaction (PCR) test results for COVID-19 were all negative. We started her treatment with prednisolone $20 \mathrm{mg} /$ day and empirical antibiotics (Fig. 1). However, her pleuritic chest pain aggravated, and transthoracic echocardiography showed minimal pericardial effusion with normal ejection fraction. Electrocardiogram showed $\mathrm{T}$ wave inversion on leads V3 and V4. Her serum levels of ferritin were $1,367 \mathrm{mg} / \mathrm{dL}$ on the 5 th day of hospitalization (HD).

To control the flare of Still's disease, we increased the dose of prednisolone to $50 \mathrm{mg} /$ day on HD 7. High-dose glucocorticoid therapy was temporarily effective in improving fever, chest pain, arthralgia, and rash for 3-4 days. However, her symptoms including pleuritic chest pain, fever, and macular rash were exacerbated again with elevated inflammatory markers on HD 13 (WBC 27,050/uL, ESR $111 \mathrm{~mm} / \mathrm{h}$, CRP $20.53 \mathrm{mg} / \mathrm{dL}$, ferritin $8,522 \mathrm{mg} / \mathrm{dL}$ ). She was treated with methylprednisolone pulse therapy ( $125 \mathrm{mg} /$ day for 3 days) followed by etanercept ( $50 \mathrm{mg}$ subcutaneous injection), methotrexate $(10 \mathrm{mg} /$ week $)$, and prednisolone $(75 \mathrm{mg} /$ day $)$. Antibiotic treatment was discontinued. This treatment approach led to transient improvement in her symptoms and laboratory findings. However, right-sided pleuritic chest pain and fever recurred with the macular rash spreading to her extremities on HD 25 (Fig. 2). Chest CT showed the presence of bilateral pleural effusion. Pleural fluid analysis were characteristic of an exudate with predominance of neutrophils as follows: $\mathrm{pH}$, 7.546; WBC, 31,000/uL (neutrophil $80 \%$, lymphocyte $20 \%$, and histiocyte $10 \%$ ); total protein, $3.9 \mathrm{~g} / \mathrm{dL}$; lactate dehydrogenase, 1,662 IU/L; adenosine deaminase, $52.3 \mathrm{IU} / \mathrm{L}$ (nor$\mathrm{mal}<83.4 \mathrm{IU} / \mathrm{L}$ ). Pleural fluid stain, cultures, cytology, and PCR test for tuberculosis were negative. Empirical antibiotics were re-administered, and the dose of prednisolone was reduced to $40 \mathrm{mg} /$ day; however, the symptoms had failed to improve. As a treatment of the refractory Still's disease, $8 \mathrm{mg}$ / $\mathrm{kg}$ of tocilizumab was administrated to HD 32 along with methotrexate and prednisolone, and symptoms and laboratory findings were significantly improved. We tapered the dose of prednisolone to $35 \mathrm{mg} /$ day and discharged the patient 7 days later. The improvement was maintained without symptoms for two months with tocilizumab ( $8 \mathrm{mg} / \mathrm{kg}$ every two weeks), methotrexate $(10 \mathrm{mg} / \mathrm{week})$ and median dose of prednisolone ( $20 \mathrm{mg} /$ day). Inflammatory markers including ESR, CRP and ferritin also remained normal.

\section{Search strategy}

According to the published guideline on narrative reviews [10], the literature search was conducted through MEDLINE/PubMed and Scopus databases up to August 2021. The search was conducted using the following queries: 'JIA' AND 'COVID-19', 'JIA' AND 'vaccination', 'COVID-19' AND 'vaccination' AND 'rheumatic disease'. We retrieved relevant results, including research articles, case reports, letters, correspondences, and reviews. We found 52 results for 'JIA' and 'COVID-19', 153 results for 'JIA' and 'vaccination', and 103 results for 'COVID-19', 'vaccination', and 'rheumatic disease'. Non-English articles were excluded from the search.

\section{Discussion}

Infection is a potential risk factor for flares in Still's disease, and vaccination can be a powerful strategy to mitigate the risk of COVID-19 infection. However, there have been some cases of disease flare following vaccination. Herein, we 


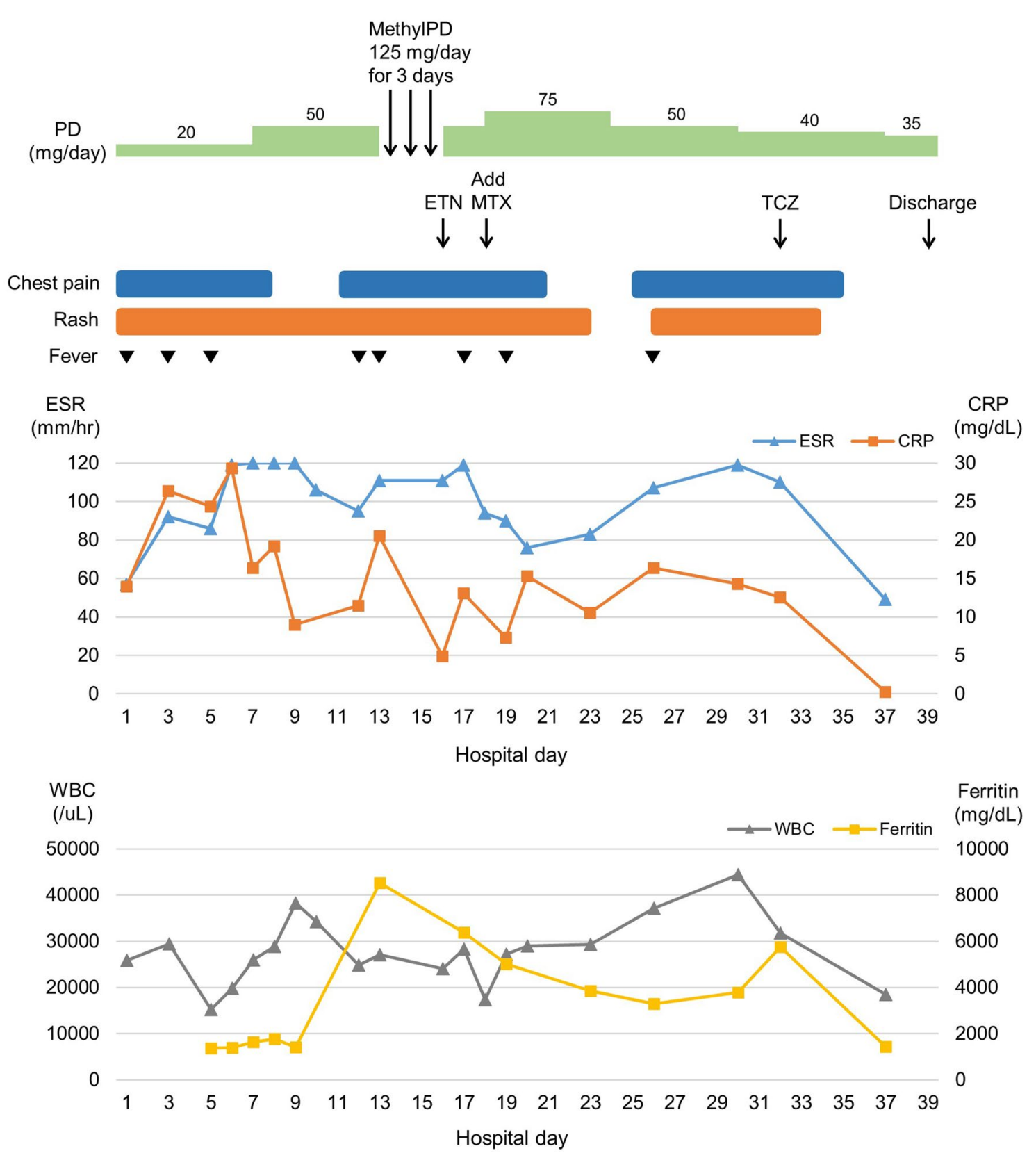

Fig. 1 Timeline of clinical and laboratory findings and treatment during the course of hospitalization in a patient with Still's disease, who underwent exacerbated disease activity following COVID-19 vac-

report an interesting case of a Still's disease flare following COVID-19 vaccination in an adult patient who had stable disease activity with etanercept therapy. Tocilizumab was an effective therapeutic agent in this case of Still's disease which underwent flaring following COVID-19 vaccination.

In our case, the patient was diagnosed with juvenileonset Still's disease at the age of 12 years. Despite low disease activity for a long time, the flare occurred following COVID-19 vaccination. In terms of JIA, there have been previous studies investigating the association between cination. $P D$ prednisolone, $E T N$ etanercept, $M T X$ methotrexate, $T C Z$ tocilizumab, ESR erythrocyte sedimentation rate, $C R P$ C-reactive protein

flares and vaccination. Australian research on routine childhood vaccination in rheumatic disease demonstrated that the risk of arthritis flares after vaccination was lower than the baseline risk [11]. A randomized trial of the safety of measles-mumps-rubella vaccination in JIA showed that it did not affect disease activity in JIA patients [12]. Likewise, in a study of JIA patients on biologic therapy, no flare was reported after influenza vaccination [13]. In contrast, Shimizu et al. described the case of a 3-year-old girl who experienced relapses of Still's disease after receiving 


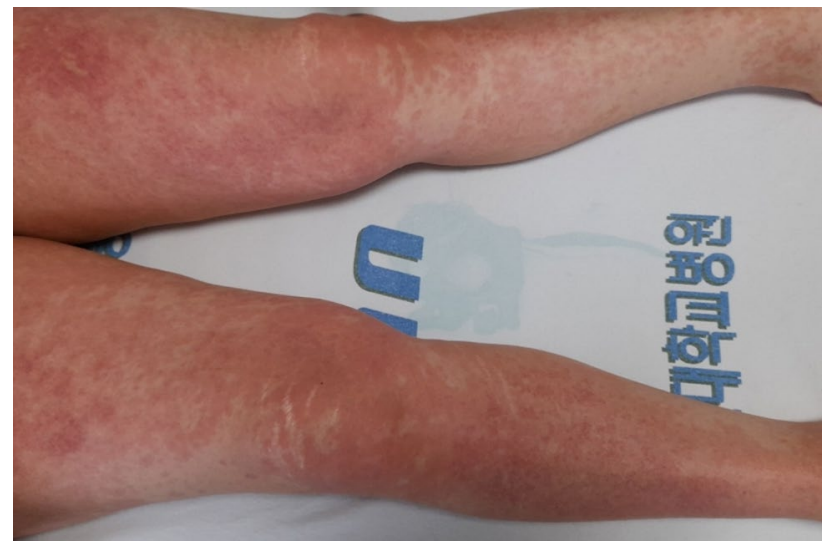

Fig. 2 Recurrent macular rash on the legs persisting despite glucocorticoid treatment in a patient with Still's disease, who underwent a disease flare following COVID-19 vaccination

influenza vaccination [14]. A report by Korematsu et al. also presented a case of Still's disease flare after rubella vaccination [15]. A prospective longitudinal study reported that influenza vaccination triggered flares in 11 of 31 patients with stable JIA [16]. These inconsistent results on the impact of vaccination on JIA flares raises questions whether COVID-19 vaccination is associated with flares of Still's disease.

Regarding COVID-19, research on the link between vaccination and disease flare is still ongoing. In a study on the efficacy and safety of the COVID-19 mRNA vaccine in chronic inflammatory disease, no patients showed a disease flare [17]. A cross-sectional study indicated that only four out of 724 patients receiving COVID-19 vaccination (the ChAdOx1 or the BBV152 vaccines) experienced the flares of arthritis and improved within 5 days [18]. In contrast, Watad et al. described 27 cases of immune-mediated disease flare or new-onset disease after COVID-19 vaccination, including systemic lupus erythematosus and Behcet's disease [19]. In our case, the patient experienced the flare one week following initial vaccination. Compared to other JIA subtypes, Still's disease is known to become less inflammatory at long-term follow-up as a 'burn-out' concept [20]. The occurrence of flares following vaccination in a patient with long-term stable disease activity suggests the possibility that COVID-19 vaccination affects the pathogenic mechanism of Still's disease. Although the association between vaccination and disease flare is not clear, this case reveals that monitoring disease activity following COVID-19 vaccination is helpful among patients with Still's disease.

The mechanism behind the occurrence of flares of Still's disease following COVID-19 vaccination is not yet known. Certain components of vaccines like pathogenic antigens may induce immune activation in autoimmunity [21]. In addition, COVID-19 infection is related to the activation of the innate immunity and the production of pro-inflammatory cytokines such as IL-1, IL-6, and TNF- $\alpha$ [22]. These inflammatory responses are similar, in terms of mechanism, to Still's disease [23]. COVID-19 vaccination may elicit production of the SARS-CoV-2 spike protein similar to those in COVID-19, and this process may also sensitize the immune system towards exacerbation of Still's disease [24, 25]. However, further research is required to delve into the association between the mechanisms of interplay between COVID-19 infection, vaccination, and Still's disease.

In this case, the flare was not controlled despite advanced therapy of methylprednisolone pulse, etanercept, and methotrexate. However, the patient improved definitely after tocilizumab therapy, which is used for treatment of Still's disease [26]. A previous report showed a successful case of tocilizumab therapy in a patient with Still's disease who experienced a relapse after influenza vaccination [14]. Similarly, no flares were reported following influenza vaccination while administering tocilizumab in Still's disease [27]. Our case suggests that tocilizumab may be a viable therapeutic option to control refractory cases of flaring in Still's disease following COVID-19 vaccination, even in patients who were otherwise well-controlled on TNF inhibitors.

Several limitations should be considered when interpreting this case study. First, it is possible that the timing of vaccination merely coincided with Still's disease's flare. The causal relationship between COVID-19 vaccination and flare cannot be clarified in this case. Therefore, vaccination should not be deferred out of concern for possible flaring of disease activity. Furthermore, Still's disease is diagnosed based on clinical presentation and upon the exclusion of other conditions. Other causes cannot be completely excluded in this case. In particular, vaccines can sometimes cause adverse events such as drug eruptions, fever, and myalgia. Because skin biopsy was not performed, the etiology of the skin lesions cannot be confirmed as necessarily arising from Still's disease itself. However, her skin rash was typical of Still's disease and usually appeared concurrently with fever. In addition, the symptoms that were not controlled by glucocorticoid administration improved with tocilizumab therapy, a known treatment for Still's disease. These details in the case may support the diagnosis of Still's disease above and over other conditions.

In conclusion, we reported a case of the flare after COVID-19 vaccination in a patient with Still's disease who maintained low disease activity with therapy of etanercept and low-dose glucocorticoid. Clinical symptoms and laboratory findings improved after tocilizumab therapy. It is clear that the benefit of vaccination outweighs the risk, considering the COVID-19 pandemic. However, this case suggests that patients with Still's disease should be carefully monitored for related symptoms following COVID-19 vaccination. Further studies are required to investigate the 
association between COVID-19 vaccination and flares of Still's disease.

Author contributions YHJ: data collection, writing and original draft. DHL: review and editing. SWC: methodology, investigation, review and editing. SJC: supervision, writing, review and editing. All authors approved the final manuscript as submitted and agree to be accountable for all aspects of the work.

\section{Declarations}

Conflict of interest The authors declare no conflicts of interest in relation to this study.

Ethical approval All procedures performed in studies involving human participants were in accordance with the ethical standards of the institutional and/or national research committee and with the 1964 Helsinki declaration and its later amendments or comparable ethical standards.

Informed consent Informed consent was obtained from the patient included in the study.

\section{References}

1. Bugatti S, Balduzzi S, De Stefano L, Manzo A, Xoxi B, Bogliolo L et al (2021) Correspondence on 'EULAR December 2020 viewpoints on SARS-CoV-2 vaccination in patients with RMDs.' Ann Rheum Dis 80:e156-e156. https://doi.org/10.1136/annrh eumdis-2021-220541

2. Rotondo C, Cantatore FP, Fornaro M, Colia R, Busto G, Rella $\mathrm{V}$ et al (2021) Preliminary data on post market safety profiles of COVID 19 vaccines in rheumatic diseases: assessments on various vaccines in use, different rheumatic disease subtypes, and immunosuppressive therapies: a two-centers study. Vaccines 9:730. https://doi.org/10.3390/vaccines9070730

3. Birck R, Kaelsch I, Schnuelle P, Flores-Suárez LF, Nowack R (2009) ANCA-associated vasculitis following influenza vaccination: causal association or mere coincidence? J Clin Rheumatol 15:289-291. https://doi.org/10.1097/RHU.0b013e3181b55fe4

4. Wang B, Shao X, Wang D, Xu D, Zhang JA (2017) Vaccinations and risk of systemic lupus erythematosus and rheumatoid arthritis: a systematic review and meta-analysis. Autoimmun Rev 16:756-765. https://doi.org/10.1016/j.autrev.2017.05.012

5. Terracina KA, Tan FK (2021) Flare of rheumatoid arthritis after COVID-19 vaccination. Lancet Rheumatol 3:e469-e470. https:// doi.org/10.1016/S2665-9913(21)00108-9

6. Rahim SEG, Lin JT, Wang JC (2021) A case of gross hematuria and IgA nephropathy flare-up following SARS-CoV-2 vaccination. Kidney Int 100:238. https://doi.org/10.1016/j.kint.2021.04. 024

7. Cohen SR, Prussick L, Kahn JS, Gao DX, Radfar A, Rosmarin D (2021) Leukocytoclastic vasculitis flare following the COVID-19 vaccine. Int J Dermatol 60:1032-1033. https://doi.org/10.1111/ijd. 15623

8. Lavery MJ, Nawimana S, Parslew R, Stewart L (2021) A flare of pre-existing erythema multiforme following BNT162b2 (PfizerBioNTech) COVID-19 vaccine. Clin Exp Dermatol 46:13251327. https://doi.org/10.1111/ced.14714

9. Krajewski PK, Matusiak Ł, Szepietowski JC (2021) Psoriasis flare-up associated with second dose of Pfizer-BioNTech
BNT16B2b2 COVID-19 mRNA vaccine. J Eur Acad Dermatol Venereol 35:e632-e634. https://doi.org/10.1111/jdv.17449

10. Gasparyan AY, Ayvazyan L, Blackmore H, Kitas GD (2011) Writing a narrative biomedical review: considerations for authors, peer reviewers, and editors. Rheumatol Int 31:14091417. https://doi.org/10.1007/s00296-011-1999-3

11. Alfayadh NM, Gowdie PJ, Akikusa JD, Easton ML, Buttery JP (2020) Vaccinations do not increase arthritis flares in juvenile idiopathic arthritis: a study of the relationship between routine childhood vaccinations on the Australian immunisation schedule and arthritis activity in children with juvenile idiopathic arthritis. Int J Rheumatol 2020:1078914. https://doi.org/10. $1155 / 2020 / 1078914$

12. Heijstek MW, Kamphuis S, Armbrust W, Swart J, Gorter S, de Vries LD et al (2013) Effects of the live attenuated measles-mumps-rubella booster vaccination on disease activity in patients with juvenile idiopathic arthritis: a randomized trial. JAMA 309:2449-2456. https://doi.org/10.1001/jama.2013.6768

13. Camacho-Lovillo MS, Bulnes-Ramos A, Goycochea-Valdivia W, Fernández-Silveira L, Núñez-Cuadros E, Neth O et al (2017) Immunogenicity and safety of influenza vaccination in patients with juvenile idiopathic arthritis on biological therapy using the microneutralization assay. Pediatr Rheumatol Online J 15:62. https://doi.org/10.1186/s12969-017-0190-0

14. Shimizu M, Ueno K, Yachie A (2012) Relapse of systemic juvenile idiopathic arthritis after influenza vaccination in a patient receiving tocilizumab. Clin Vaccine Immunol 19:1700-1702. https://doi.org/10.1128/CVI.00309-12

15. Korematsu S, Miyahara H, Kawano T, Yamada H, Akiyoshi K, Sato K et al (2009) A relapse of systemic type juvenile idiopathic arthritis after a rubella vaccination in a patient during a long-term remission period. Vaccine 27:5041-5042

16. Toplak N, Subelj V, Kveder T, Cucnik S, Prosenc K, TrampusBakija A et al (2012) Safety and efficacy of influenza vaccination in a prospective longitudinal study of 31 children with juvenile idiopathic arthritis. Clin Exp Rheumatol 30:436-444

17. Geisen UM, Berner DK, Tran F, Sümbül M, Vullriede L, Ciripoi $M$ et al (2021) Immunogenicity and safety of anti-SARS-CoV-2 mRNA vaccines in patients with chronic inflammatory conditions and immunosuppressive therapy in a monocentric cohort. Ann Rheum Dis 80:1306-1311. https://doi.org/10.1136/annrh eumdis-2021-220272

18. Cherian S, Paul A, Ahmed S, Alias B, Manoj M, Santhosh AK et al (2021) Safety of the ChAdOx1 nCoV-19 and the BBV152 vaccines in 724 patients with rheumatic diseases: a post-vaccination cross-sectional survey. Rheumatol Int 41:1441-1445. https://doi.org/10.1007/s00296-021-04917-0

19. Watad A, De Marco G, Mahajna H, Druyan A, Eltity M, Hijazi $\mathrm{N}$ et al (2021) Immune-mediated disease flares or new-onset disease in 27 subjects following mRNA/DNA SARS-CoV-2 vaccination. Vaccines 9:435. https://doi.org/10.3390/vaccines90 50435

20. Packham JC, Hall MA (2002) Long-term follow-up of 246 adults with juvenile idiopathic arthritis: functional outcome. Rheumatology 41:1428-1435. https://doi.org/10.1093/rheumatology/41.12. 1428

21. Segal Y, Shoenfeld Y (2018) Vaccine-induced autoimmunity: the role of molecular mimicry and immune crossreaction. Cell Mol Immunol 15:586-594. https://doi.org/10.1038/cmi.2017.151

22. Ahmed-Hassan H, Sisson B, Shukla RK, Wijewantha Y, Funderburg NT, Li Z et al (2020) Innate immune responses to highly pathogenic coronaviruses and other significant respiratory viral infections. Front Immunol 11:1979. https://doi.org/10.3389/ fimmu.2020.01979

23. Kessel C, Hedrich CM, Foell D (2020) Innately adaptive or truly autoimmune: is there something unique about systemic juvenile 
idiopathic arthritis? Arthritis Rheumatol 72:210-219. https://doi. org/10.1002/art.41107

24. Zhao Y, Kuang M, Li J, Zhu L, Jia Z, Guo X et al (2021) SARSCoV-2 spike protein interacts with and activates TLR41. Cell Res 31:818-820. https://doi.org/10.1038/s41422-021-00495-9

25. Mellins ED, Macaubas C, Grom AA (2011) Pathogenesis of systemic juvenile idiopathic arthritis: some answers, more questions. Nat Rev Rheumatol 7:416-426. https://doi.org/10.1038/nrrheum. 2011.68

26. De Benedetti F, Brunner HI, Ruperto N, Kenwright A, Wright S, Calvo I et al (2012) Randomized trial of tocilizumab in systemic juvenile idiopathic arthritis. N Eng J Med 367:2385-2395. https:// doi.org/10.1056/NEJMoa1112802
27. Shinoki T, Hara R, Kaneko U, Miyamae T, Imagawa T, Mori M et al (2012) Safety and response to influenza vaccine in patients with systemic-onset juvenile idiopathic arthritis receiving tocilizumab. Mod Rheumatol 22:871-876. https://doi.org/10.1007/ s10165-012-0595-z

Publisher's Note Springer Nature remains neutral with regard to jurisdictional claims in published maps and institutional affiliations. 